\title{
Current options and future possibilities for the systemic treatment of hepatocellular carcinoma
}

\author{
Jean-Luc Raoul*,1, Jean-Sébastien Frenel${ }^{1}$, Judith Raimbourg ${ }^{1}$ \& Marine Gilabert ${ }^{2}$ \\ ${ }^{1}$ Department of Medical Oncology, Institut de Cancérologie de l'Ouest, Nantes 44805 Saint-Herblain, France \\ ${ }^{2}$ Department of Medical Oncology, Institut Paoli-Calmettes, Cedex 9, Marseille 13000, France \\ *Author for correspondence: jean-luc.raoul@ico.unicancer.fr
}

\begin{abstract}
Most hepatocellular carcinoma patients could not benefit from or experience disease recurrence after curative treatments. In 2007 sorafenib demonstrated efficacy in first line treatment of advanced hepatocellular carcinoma. After a decade of negative trials, in early 2019 we now have another tyrosine kinase inhibitor available in first line, lenvatinib, three other targeted therapies in second line post-sorafenib (regorafenib, cabozantinib and ramucirumab) and promising data from two immunotherapies (nivolumab and pembrolizumab). Unfortunately, no biomarkers have been identified to help guide our choice. In this short review we summarize the results of these different therapies and propose a therapeutic algorithm based on subgroup analysis. It is most likely that we will not have head-to-head comparisons in second line trials.
\end{abstract}

First draft submitted: 8 January 2019; Accepted for publication: 14 February 2019; Published online: 4 June 2019

Keywords: cabozantinib • lenvatinib • nivolumab • pembrolizumab • ramucirumab • regorafenib • sorafenib

Hepatocellular carcinoma (HCC) is a common and severe cancer which develops on an underlying chronic liver disease, usually at the stage of cirrhosis [1]. HCC is on the rise in Western countries, due in particular to the growing incidence of metabolic syndrome [2,3]. Worldwide, hepatitis B and C virus infections and alcoholism represent the major etiologies of chronic liver disease [4]. Unfortunately, despite the development of surveillance allowing early diagnosis, many cases present an advanced stage as defined by the Barcelona Clinic of Liver Cancer (BCLC) staging system [5]. This system differentiates prognostic classes based on severity of the underlying liver cirrhosis, performance status (PS) and tumor extension. Advanced stage corresponds to patients who have preserved liver function and either impaired PS 1 or 2, or extrahepatic spread or venous involvement. These patients, as well as those from intermediate stages in whom transarterial chemoembolization failed, are candidates for systemic treatments. In 2007, the first demonstration of efficacy of a systemic treatment was obtained in two Phase III trials comparing sorafenib and placebo, conducted simultaneously in Western countries (SHARP trial) [6] and in the Asia-Pacific region (AP trial) [7]. After a long period of negative trials despite inclusion of thousands of patients [8], the last 2 years have been more productive, with demonstrations of efficacy of four drugs (lenvatinib in first line, regorafenib, cabozantinib and ramucirumab postsorafenib) [9-13] and attractive data from Phase II immunotherapy trials (nivolumab in first and second line and pembrolizumab in second line) [14,15]; results from Phase III trials with these checkpoint inhibitors are eagerly awaited. In this review we will summarize the indications of these drugs with positive or promising results and propose a treatment algorithm. Obviously, new data from Phase III trials with checkpoint inhibitors could dramatically modify these suggestions.

\section{First line treatment}

Sorafenib

Sorafenib is an oral multikinase inhibitor, targeting mainly VEGFR2, PDGFR, RAF, RAS and KIT. The SHARP trial [6], a multicenter, randomized, double-blind, placebo-controlled, Phase III trial, included 602 patients: 299 in 
the sorafenib arm $(800 \mathrm{mg} /$ day per os) and 303 in the placebo arm. Stratification factors were: region, PS, vascular invasion and/or extrahepatic spread. Major inclusion criteria were: histologically confirmed advanced stage HCC, naive to systemic treatment or intermediate stage patients who progressed after locoregional treatments, PS 2 or less, well compensated liver function (Child-Pugh A). Treatment was continued until either radiologic and symptomatic progression or unacceptable toxicity. The primary outcomes were overall survival (OS) and time to symptomatic progression; secondary outcomes were time to radiologic progression, disease-control rate and safety. The study was stopped for efficacy after the second planned interim analysis. Population characteristics were well balanced across both arms; $82 \%$ had advanced stage, 53\% extrahepatic spread and 36\% macroscopic vascular invasion. Median overall survival (mOS) was significantly better in the sorafenib arm (10.7 months) than in the placebo arm (7.9 months; HR: 0.69 ; 95\% CI: $0.55-0.87$; p < 0.001). Survival rates at 1 year were respectively 44 and $33 \%$ for sorafenib and placebo. There was no difference in time to symptomatic progression but time to progression was significantly better with sorafenib ( 5.5 vs 2.8 months; $\mathrm{p}<0.001)$. These results were obtained despite a very poor response rate ( $2 \%$ with sorafenib; $1 \%$ with placebo), but the disease control rate was higher with sorafenib ( 43 vs $32 \%)$. The median duration of treatment was 5.3 months in the sorafenib arm and 4.3 months in the placebo arm. In the sorafenib arm $76 \%$ of the patients received more than $80 \%$ of the planned dose. The major toxicities were constitutional syndrome, diarrhea, dermatologic events and anorexia. Dose reductions and dose interruptions due to adverse events were observed in 26 and $44 \%$ of patients on sorafenib. No deaths were considered related to sorafenib.

The Asia-Pacific trial included 271 patients [7] from China, Taiwan and South Korea. Randomization was stratified by the presence or absence of macroscopic venous invasion or extrahepatic spread, PS and geographic region and randomized in a 2:1 ratio between sorafenib and placebo. The mOS was better in the sorafenib arm than in the placebo arm ( 6.5 vs 4.2 months; HR: $0.68 ; 95 \%$ CI: $0.50-0.93 ; \mathrm{p}=0.014)$. Time to tumor progression was better in the sorafenib arm. The response rate was low (3 vs $1 \%$ ). The safety profile was similar to that seen in the SHARP trial.

In 2017 a pooled analysis of these two trials focused on prognostic factors and predictors of sorafenib benefit [16]. Strong prognostic factors for poorer survival were: macroscopic venous invasion, high AFP levels and a high neutrophil-to-lymphocyte ratio. Sorafenib benefit was consistently observed in all subgroups but was greater in patients without extrahepatic spread, with hepatitis $C$ virus infection and low neutrophil-to-lymphocyte ratio.

Once under treatment, patients who developed early (first 60 days) dermatologic events had a better mOS than those who did not present these reactions (18.2 vs 10.1 months) [17]. In a retrospective analysis of 1119 Spanish HCC patients treated with sorafenib [18], 12 had a complete response according to RECIST 1.1, all but one of whom had an early dermatologic reaction; their mOS was 85.8 months!

Prognostic factors of survival after sorafenib failure are not well known. Some are intuitive and have been confirmed in retrospective analyses [19,20], such as Child-Pugh classification, PS, time to progression on sorafenib, while others have emerged from these analyses: AFP serum level, macrovascular invasion (which has a worse prognosis than extrahepatic metastases), pattern of progression (particularly poor prognosis for patients who progressed with new extrahepatic lesions or vascular invasion) [20-22].

\section{Lenvatinib}

Lenvatinib is an oral multityrosine kinase inhibitor targeting VEGFR1-3, FGFR1-4, PDGFR, RET and KIT [23,24]. An open-label, Phase III, multicenter, noninferiority trial (noninferiority margin set at 1.08) compared sorafenib with lenvatinib [9], in a 1:1 ratio, in first line systemic treatment for patients with advanced stage HCC confirmed histologically or cytologically (REFLECT trial). Stratification factors were: region, macroscopic portal vein invasion/extrahepatic spread or both, PS and bodyweight. Inclusion criteria were: BCLC B or C, Child-Pugh class A, PS 0-1 with correct biological parameters. Exclusion parameters were: more than $50 \%$ of the liver volume occupied by the tumor, obstruction of the bile duct, invasion of the main portal vein. The lenvatinib dose depended on bodyweight, $12 \mathrm{mg} /$ day if over $60 \mathrm{~kg}$ and $8 \mathrm{mg} /$ day if under; the sorafenib dose was $800 \mathrm{mg} /$ day. The primary outcome was OS and secondary end points were progression-free survival, quality of life and response rate. A total of 954 patients were included, 478 in the lenvatinib arm and 476 in the sorafenib arm. Baseline characteristics were similar except for $\mathrm{C}$ virus infection and baseline AFP $<200 \mathrm{ng} / \mathrm{ml}$, more frequent in the sorafenib arm. The mOS for lenvatinib was not inferior (HR: 0.92; 95\% CI: 0.79-1.06) to sorafenib (13.6 vs 12.3 months). However, median progression-free survival (mPFS), time to progression and response rates (mRECIST; masked independent imaging: 40.6 vs $12.4 \%$; RECIST 1.1: 18.8 vs $6.5 \%$ ) were significantly better with lenvatinib. Subgroup analysis 
Table 1. Main data from unplanned subgroup analyses from the three placebo-controlled Phase III trials of regorafenib, cabozantinib and ramucirumab in second line post-sorafenib.

\begin{tabular}{|c|c|c|c|}
\hline & $\begin{array}{l}\text { Regorafenib } \\
(n=573)\end{array}$ & $\begin{array}{l}\text { Cabozantinib }{ }^{11} \\
(n=707)\end{array}$ & $\begin{array}{l}\text { Ramucirumab }{ }^{12} \\
(n=292)\end{array}$ \\
\hline OS: HR $(95 \% \mathrm{Cl})$ & $0.63(0.50-0.79)$ & $0.76(0.63-0.92)$ & $0.74(0.56-0.99)$ \\
\hline Males/females & $++/+$ & $++/+$ & $++/-$ \\
\hline Age: $</>65$ years & $++/+$ & $+/++$ & $+/++$ \\
\hline PS $0 / 1$ & $++/+$ & $++/+$ & $+/+$ \\
\hline Asia/West & $++/++$ & $0 /++$ & $+/+$ \\
\hline HBV: yes & ++ & ++ & + \\
\hline HCV: yes & + & - & + \\
\hline AFP: low/high & $++/++$ & $+/++$ & $-/++$ \\
\hline MVI: yes/no & $++/++$ & $+/+$ & $(-) /++$ \\
\hline EHS: yes/no & $++/ 0$ & $++/ 0$ & $++/+$ \\
\hline
\end{tabular}

EHS: Extrahepatic spread; HBV: Hepatitis B virus infection; HCV: Hepatitis C virus infection; HR: Hazard ratio; MVI: Macroscopic venous invasion; OS: Overall survival; PS: Performance status.

of OS did not demonstrate any difference or trend between sorafenib and lenvatinib for younger ( $<65$ years old), western or PS 1 patients, or in case of absence of macroscopic vein invasion or extrahepatic metastases. Lenvatinib appears (but not significantly) to be more efficient in hepatitis B virus infection (HBV) or Asia-Pacific patients, in case of portal vein invasion or extrahepatic spread, and for those with high AFP (>200 ng/ml; HR: 0.78; 95\% CI: 0.63-0.98). In none of the subgroups did sorafenib do better than lenvatinib. Lenvatinib was superior to sorafenib in all subgroups regarding progression-free survival. Lenvatinib gave more frequent adverse events than sorafenib but this can be explained by significantly longer treatment duration (median: 5.7 vs 3.7 months). The most common grade III adverse events differed between the two drugs: hypertension, weight loss with lenvatinib, skin toxicity and diarrhea with sorafenib. The percentage of patients requiring dose interruption, modification or withdrawal as well as the dose intensity was similar in both arms (88\% with lenvatinib and $83 \%$ with sorafenib). Fatal adverse events were observed in 11 patients with lenvatinib and four with sorafenib. The deterioration in some quality of life parameters was observed earlier with sorafenib.

Therefore, from a standpoint of OS, lenvatinib was noninferior to sorafenib but did better on response rate, progression-free survival and time to progression; the safety profile was similar to that observed with sorafenib but lenvatinib delayed the deterioration in quality of life scores. Its impact on survival, compared with sorafenib, seems promising in $\mathrm{HBV}$ and high AFP patients.

There are no biomarkers to guide the choice between sorafenib and lenvatinib.

Lenvatinib (Lenvima ${ }^{\circledR}$ ) was approved in 2018 by Japanese authorities, the US FDA and the EMA in first line treatment of unresectable advanced HCC.

\section{Second line treatments}

Regorafenib

Regorafenib is an oral multityrosine kinase inhibitor targeting VEGFR 1-3, PDGFR, FGFR, TIE2, KIT, RET and BRAF. Its molecular profile and pharmacological activity are different from those of sorafenib [25].

Efficacy of regorafenib versus placebo (Table $1 \& 2$ ) was demonstrated in an international, randomized [10], placebo-controlled trial (RESORCE trial), comparing regorafenib (379 patients) to placebo (194 patients), in a 2:1 ratio, in Child-Pugh A, PS 0-1 patients who progressed under but tolerated sorafenib $(\geq 400 \mathrm{mg} /$ day at least 20 of the 28 days before discontinuation). Regorafenib was given per os at $160 \mathrm{mg} /$ day for 3 weeks on, 1 week off, until disease progression, clinical progression or symptomatic progression. Stratification factors were: region, macrovascular invasion, extrahepatic spread, AFP level $(/ 400 \mathrm{ng} / \mathrm{ml})$ and PS $(0 / 1)$. The primary end point was OS.

Baseline characteristics were similar between the two arms; duration of sorafenib treatment in first line was 7.8 months in both arms.

The $\mathrm{mOS}$ was better in the regorafenib group: 10.6 versus 7.8 months (HR: $0.63 ; 95 \%$ CI: $0.50-0.79$ ). This improvement in mOS was observed in all subgroups and was particularly important in certain subgroups: males, 


\begin{tabular}{|c|c|c|c|c|c|c|}
\hline & Regorafenib $^{10}$ & Cabozantinib $^{11}$ & Ramucirumab $^{12}$ & Pembrolizumab $^{14}$ & Nivolumab $^{13}$ & Nivolumab $^{25}$ \\
\hline & $\mathrm{Ph}$ III & Ph III & $\mathrm{Ph}$ III & Ph II & Ph II & Doc FDA \\
\hline $\mathrm{N}$ & 379 & 470 & 197 & 104 & 57 & 154 \\
\hline Intol/progr \% & $0 / 100$ & $? / ?$ & $16 / 84$ & $20 / 80$ & $0 / 100$ & $? / ?$ \\
\hline Sorafenib (duration) & $7.8 \mathrm{~m}$ & $5.3 \mathrm{~m}$ & UK & 6.8 & UK & UK \\
\hline EHS (\%) & 70 & 79 & 71.6 & 64 & UK & 71 \\
\hline MVI (\%) & 29 & 27 & 35.5 & 17 & UK & 29 \\
\hline \% AFP high (limit) & $43(400)$ & $41(400)$ & $100(400)$ & $41(200)$ & UK & $37(400)$ \\
\hline RR (RECIST 1.1) & $7 \%$ & $4 \%$ & $4.6 \%$ & $17 \%$ & $21 \%$ & $14.3 \%$ \\
\hline DCR (\%) & 66 & 64 & 59.9 & 62 & 61 & UK \\
\hline PD (\%) & 22 & 21 & 33.5 & 33 & 32 & - \\
\hline TTP (months) & 3.2 & UK & - & 4.9 & - & - \\
\hline PFS (months) & 3.1 & 5.2 & 2.8 & 4.9 & 4.0 & - \\
\hline mOS (months) & 10.6 & 10.2 & 8.5 & 12.9 & 13.2 & - \\
\hline mOS: HR & 0.63 & 0.76 & 0.71 & - & - & - \\
\hline Tt (duration) & 3.6 & 3.8 & UK & 4.2 & UK & UK \\
\hline Response (duration) & 3.5 & UK & UK & $9+$ & $?$ & $12+?$ \\
\hline Cessation/tox (\%) & 25 & 16 & 10.7 & 5 & 4 & - \\
\hline Dose intensity (\%) & 90 & 60 & 98 & UK & UK & UK \\
\hline
\end{tabular}

DCR: Disease control rate; EHS: Extrahepatic spread; HR: Hazard ratio; Intol: Intolerant to sorafenib; m: Months; mOS: Median overall survival; MVI: Macroscopic venous invasion; PD: Progressive disease; PFS: Progression-free survival; Progr: Progressor on sorafenib; RECIST 1.1: Response evaluation criteria in solid tumors version 1.1; RR: Response rate; tox: Toxicity; Tt: Treatment; TTP: Time to progression; UK: Unknown.

PS 0, Child-Pugh A5, metastatic patients and those with B virus infection and no alcohol use. Response rate (mRECIST) was 11 versus $4 \%$ and disease control rate was 65 versus $36 \%$.

Median times on treatment were 3.6 months with regorafenib and 1.9 with placebo. Severe adverse events (SAE) were more frequent in the regorafenib group (hypertension, hand-foot syndrome, fatigue and diarrhea). Severe (Grade III) dermatologic toxicity of regorafenib was observed in $13 \%$ of the population, similar to what was observed with sorafenib first line. Nevertheless, no clinically meaningful difference in quality of life was noted between the two arms. It is important to note that the clinical benefit was observed regardless of the last dose of sorafenib (full dose or $400 \mathrm{mg} /$ day) or the time to progression on sorafenib [26].

Regorafenib (Stivarga ${ }^{\circledR}$ ) received FDA and EMA therapeutic indication in 2017 for the treatment of adult patients with HCC who have been previously treated with sorafenib.

\section{Cabozantinib}

Cabozantinib, an oral multityrosine kinase inhibitor targeting VEGFR1-3, MET and AXL [27], was compared (60 mg per os once per day) in a double-blind Phase III trial (CELESTIAL trial) to placebo [11] in a 2:1 ratio (Table 1, 2). Inclusion criteria were: histologically proven HCC, Child-Pugh A, PS $0-1$, with adequate hematologic and renal function; patients must have progressed after systemic treatment including sorafenib but could have received up to two systemic treatments before inclusion. Stratification factors were: etiology, geographic area, extrahepatic spread and/or macrovascular invasion. The primary end point was OS. Patient characteristics in the two arms (470 patients treated with cabozantinib and 237 with placebo) were well balanced; $25 \%$ of the patients came from Asia, more than $50 \%$ were PS 0 , more than a third had B virus infection, $78 \%$ had extrahepatic spread and $30 \%$ macrovascular invasion (and $85 \%$ had one or both); $27 \%$ had previously received two lines, sorafenib in all cases, the other line was essentially systemic chemotherapy or investigational agents. The trial was stopped at the second interim analysis, after inclusion of 707 patients for efficacy. The mOS was statistically better (HR: 0.76; 95\% CI: $0.63-0.92)$ in the cabozantinib versus placebo arm (10.2 vs 8.0 months). The mPFS was significantly longer with cabozantinib than with placebo (5.2 vs 1.9 months; $\mathrm{p}<0.001)$. The objective response rate (RECIST 1.1$)$ was $4 \%$ in the cabozantinib arm and less than $1 \%$ in the placebo arm and the disease control rate was 64 versus $33 \%$. The median duration of treatment was 3.8 months with cabozantinib and 2.0 months with placebo. Dose reductions were more frequent with cabozantinib (62\%) and the median average daily dose was $35.8 \mathrm{mg}$ for cabozantinib (close to that seen in renal cell cancer) and $58.9 \mathrm{mg}$ for placebo. The most common Grade III-IV severe adverse events in 
the cabozantinib arm were those of other anti-VEGFR tyrosine kinase inhibitors: palmar-plantar erythrodyesthesia $(17 \%)$, hypertension $(16 \%)$, fatigue $(10 \%)$ and diarrhea $(10 \%)$; increase in transaminases was noted in $12 \%$. Grade $\mathrm{V}$ adverse events considered as drug-related were reported in six patients on cabozantinib and one on placebo. Subgroup analysis of PFS consistently favored cabozantinib. Results on OS were variable; benefit was statistically significant for patients over 65 years old, males, PS 0 , non-Asian, AFP $\geq 400 \mathrm{ng} / \mathrm{ml}$, with extrahepatic spread and $B$ virus infection and patients who received only sorafenib previously (by definition progressors on sorafenib); no difference was noted with placebo for Asian patients, for those who did not have extrahepatic spread and for those with $\mathrm{C}$ virus infection.

Cabozantinib (Cabometyx ${ }^{\circledR}$ ) has been approved by the FDA and the EMA as monotherapy for the treatment of HCC in adults who have previously been treated with sorafenib.

\section{Ramucirumab}

Ramucirumab is a recombinant IgG1 monoclonal antibody that specifically binds to VEGFR-2 [28]. In a large randomized, double blind, multicenter Phase III trial versus placebo (ratio 1:1) as second line treatment after sorafenib (REACH trial) including 565 patients [13,29], the difference in mOS between the two arms $(9.2$ months for patients treated with ramucirumab and 7.6 months for the placebo arm) did not reach significance (HR: 0.87; $95 \%$ CI: 0.72-1.05), but in the subgroup analysis of OS, the prespecified subgroup of patients having a high AFP level $(>400 \mathrm{ng} / \mathrm{ml} ; \mathrm{n}=250)$ had significantly better OS on ramucirumab (7.8 vs 4.2 months; HR: $0.67 ; 95 \%$ CI: $0.51-0.90)$.

Subsequently, a second randomized controlled trial versus placebo (Table $1 \& 2$ ), limited to the subgroup of patients with high AFP (>400 ng/ml), was conducted. This trial (REACH 2 trial) [12], including 292 patients, gave positive results with a gain in mOS from 7.3 up to 8.5 months with ramucirumab (HR: 0.71 ; $95 \%$ CI: $0.53-$ 0.95). In all subgroups (except female), ramucirumab did (not significantly) better than placebo; the difference was significantly in favor of ramucirumab for males, patients older than 65 years, with extra-hepatic metastases and for those without macrovascular invasion. Progression-free survival was significantly better with ramucirumab; response rate (4.6 vs $1.1 \%$ ) was not different from that seen with placebo. By pooling the two groups of patients with high AFP values under ramucirumab in REACH and REACH-2, the gain in mOS was 3.1 months (8.1 vs 5.0 months; HR: 0.69; 95\% CI: 0.57-0.84).

Safety was poor in patients with decompensated cirrhosis. In the REACH study, initially, Child-Pugh B patients could be included, but due to an increase in toxicity in the ramucirumab arm, the trial was amended to exclude these patients. In these patients, edema, anorexia, asthenia and nausea were more frequent in the ramucirumab arm than in the placebo arm [30].

\section{Nivolumab}

Nivolumab, a fully humanized monoclonal antibody, is a PD-1 check-point inhibitor. In 2017, the results of an open-label, noncomparative, Phase I/II dose escalation and dose expansion trial in patients with HCC (CheckMate 040) were reported [14]. The dose escalation phase ( $\mathrm{n}=48$; Child-Pugh A and B7) differentiated patients who were uninfected or infected with hepatitis B virus infection (HCV) or HBV (concomitantly receiving an anti-HBV treatment). The dose of $3 \mathrm{mg} / \mathrm{kg}$ every 2 weeks was chosen for the dose expansion phase due to the excellent tolerance (including good hepatic tolerance) in the three subgroups.

The dose expansion phase $(\mathrm{n}=214$; Child-Pugh A) comprised four groups: uninfected untreated or intolerant to sorafenib $(n=56)$, uninfected progressor $(n=57), H C V$-infected $(n=50)$ and HBV-infected $(n=51)$. Patients were Child-Pugh A, PS 0-1, 29\% had vascular invasion and 37\% had AFP $>400 \mathrm{ng} / \mathrm{ml}$. The safety profile was excellent in all groups, and the most common cause of treatment discontinuation was progression. The response rate (RECIST 1.1) was 20\% (95\% CI: 15-26\%), including three complete responses and responses were durable (median duration of response: 9.9 months). The disease control rate was 64\% (95\% CI: $58-71 \%$ ). Tolerance was very good, with only $4 \%$ of patients discontinuing nivolumab due to drug toxicity. Grade III/IV treatmentemergent toxicities were seen in $19 \%$ of patients and treatment-emergent severe adverse events in $4 \%$ (rash, fatigue and diarrhea); immune-mediated hepatitis was infrequent. In the uninfected progressor group, the response rate was $12 \%$ and the mOS was 13.2 months. The response rate among the 145 patients previously treated with sorafenib (Table 2) was 19\%, including five complete responses. PD-L1 expression was retrospectively assessed by immunohistochemistry in 174 patients: with a cut-off value of PD-L1 membrane expression on at least $1 \%$ of tumor cells, $20 \%$ of the patients were considered as positive but without any predictive value on response rate. 
Based on these impressive results, nivolumab has been registered in the USA [31], Japan and some other countries in this indication of HCC previously treated with sorafenib. Results of the Phase III trial comparing first line treatment with sorafenib versus nivolumab (CheckMate 459) are eagerly awaited. Of note, at the ESMO 2018 meeting, a real-world series [32] of 76 Asian patients (but a quarter were Child-Pugh B) reported a disappointing response rate of $7.9 \%$ and a disease control rate of $39.5 \%$; tolerance was good.

\section{Pembrolizumab}

Pembrolizumab, an anti-PD1 monoclonal antibody, was tested in an open-label Phase II trial in patients with advanced HCC previously treated with sorafenib (only one systemic previous line accepted) (KEYNOTE-224) [15] at $200 \mathrm{mg}$ intravenously every 3 weeks (Table 2). The study population comprised 104 patients, PS $0-1$, ChildPugh A in $94 \%$, BCLC C in $76 \%$ and B in $24 \%$, who previously interrupted sorafenib due to failure (80\%) or intolerance (20\%). Most patients had alcohol-related disease, only $17 \%$ had macrovascular invasion, and $41 \%$ had a baseline AFP $>200 \mathrm{ng} / \mathrm{ml}$. The median duration of sorafenib treatment before inclusion was 6.8 months. The median duration of pembrolizumab treatment was 4.2 months and causes of treatment discontinuation were progression in $57 \%$ and toxicity in $23 \%$. In this second line cohort, the objective response was $17 \%$ (95\% CI: $11-26 \%$ ), and the disease control rate was $62 \%$ (95\% CI: $52-71 \%$ ). The median time to progression was 4.9 months, and the median duration of response was not reached, $77 \%$ of the responders showed a response for at least 9 months; mOS was 12.9 months. The safety profile seemed good but one patient died from esophagitis and immune-mediated hepatitis occurred in three patients. PD-L1 expression, assessed retrospectively in 52 patients using a combined positive score and a tumor proportion score, was associated with tumor response. A Phase III trial in second line postsorafenib is ongoing (KEYNOTE-240).

Based on these results, on 9 November 2018, the FDA accelerated approval of pembrolizumab [31] for HCC patients previously treated with sorafenib.

In conclusion, we now have two options for first line systemic treatment in advanced unresectable HCC: sorafenib and lenvatinib (Figure 1). Only sorafenib has been tested in PS 2 patients and in those with large tumor and thrombosis of the portal trunk. For other patients, lenvatinib gives a better response rate and time to progression (but without impact on survival) and was noninferior to sorafenib in OS; in subgroup analyses (unplanned, with questionable conclusion), lenvatinib seemed to improve mOS for patients with $\mathrm{B}$ virus infection, those with extrahepatic spread or macroscopic venous invasion and those with high AFP level.

In second line, all the currently available drugs have been tested versus placebo (Figure 1). Intertrial comparisons are not only difficult but also 'forbidden'. Regorafenib cannot be prescribed to patients who poorly tolerated sorafenib. For this population of sorafenib-intolerant patients, ramucirumab can be proposed for those with AFP $>400 \mathrm{ng} / \mathrm{ml}$ but the results are poor if they have a portal vein thrombosis. Cabozantinib is a good option in second line postsorafenib and more questionable later as its efficacy is minimal for patients who received two previous regimens. For patients who progressed on sorafenib but had good tolerance, the three drugs (regorafenib, cabozantinib and ramucirumab) can be considered. As these drugs have only been compared with placebo, there are no clear data to guide the choice and treatment decisions can only be based on experience and on unplanned subgroup analyses. Regorafenib improved OS in all subgroups except in patients without extrahepatic metastases, in case of alcohol abuse and in females. The impact of cabozantinib on OS was significant in patients older than 65, PS 0, high AFP (>400 ng/ml), with extrahepatic spread and HBV infection, and more questionable in patients without metastases, with HCV infection and from Asia. Ramucirumab does not appear to benefit patients who are female or who have macrovascular invasion. It is obvious that a direct comparison between these different drugs would be of major importance, but. . .

As lenvatinib is now recognized as a first-line treatment, the question of what to use in second line after lenvatinib is open, since all second-line trials have been performed after sorafenib. At the 2019 ASCO GI meeting, a post hoc analysis of subsequent medications after lenvatinib responders from the REFLECT study showed that a third of lenvatinib patients could receive a second line, sorafenib in most cases [33].

Regarding immunotherapy, the results and particularly the impressive duration of response are very attractive but nothing more can be gleaned from these early phases and we have to wait for the data from randomized Phase III trials, versus sorafenib in first line and, unfortunately, versus placebo in second line, to determine their indication. In first and second line, prices will matter and the price of these drugs will have to be evaluated in the light of their clinical benefit according to the ESMO-MCBS (Magnitude of Clinical Benefit Scale) [34]. 


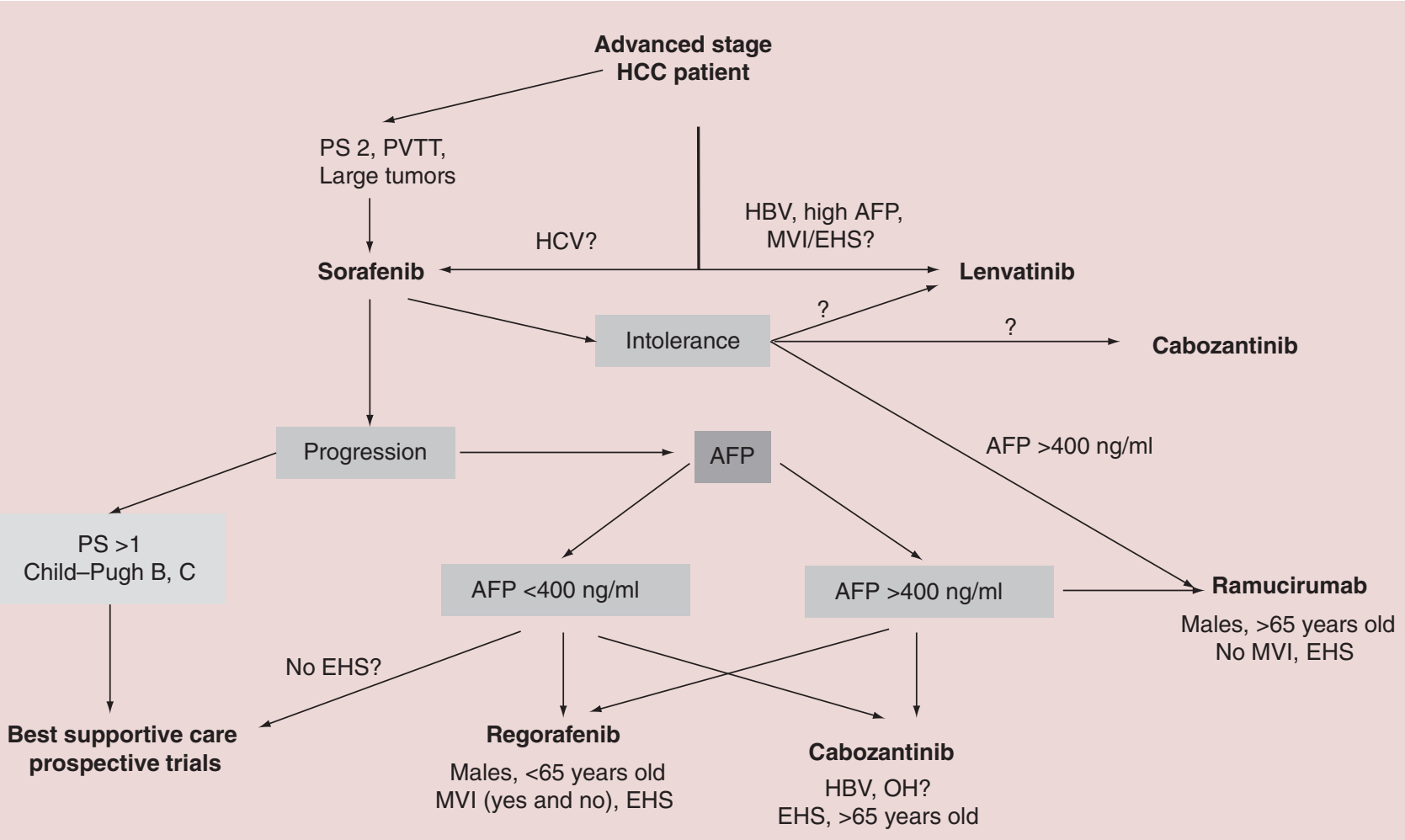

Figure 1. Proposed treatment algorithm of medical treatment in hepatocellular patients (hepatocellular carcinoma). Immunotherapies are not on this algorithm due to absence of Phase III trials and this figure will have to be redrawn after publication of trial results. EHS: Extrahepatic spread; HBV: Hepatitis B virus infection; HCV: Hepatitis C virus infection; MVI: Macroscopic venous invasion; OH: Ethanol abuse; PS: Performance status; PVT: Portal vein trunk thrombosis.

\section{Conclusion}

We currently have five drugs with demonstrated efficacy in the systemic treatment of HCC: two in first-line (sorafenib, lenvatinib) and three in second-line post-sorafenib (regorafenib, cabozantinib and ramucirumab). Immunotherapies (nivolumab and pembrolizumab) gave impressive results in early phases and results from Phase III trials are eagerly awaited.

In first line, lenvatinib demonstrated noninferiority when compared with sorafenib but secondary end points (progression-free survival, response rate and time to progression) favored lenvatinib. Toxicity was similar in both arms.

In second line after sorafenib, regorafenib, only in patients who tolerated sorafenib, did better than placebo with a survival gain of close to 3 months; tolerance in this population was correct. Cabozantinib improved OS by almost 2 months, regardless of tolerance to sorafenib; dose reductions were frequent and the safety profile was close to that of sorafenib. Ramucirumab, a pure antiangiogenic drug, conferred a survival gain of more than two months (more than 3 months by pooling the results of two studies) compared with placebo in patients with high AFP level, with good tolerance in patients with well compensated cirrhosis.

Nivolumab (in first and second line) and pembrolizumab (in second line) gave impressive results and particularly long-lasting tumor responses with excellent tolerance. Results from ongoing Phase III trials are pending.

Unfortunately, so far no biomarkers as predictors of benefit are available to guide our choice and additional research is needed.

\section{Future perspective}

After a decade with sorafenib as the only systemic treatment of HCC, four drugs have now entered this field and two are knocking at the door. In the near future two main problems need to be solved: how to choose between these drugs in first- and second-line settings? Studies dedicated to biomarkers predictive of response are desperately 
needed but also trials comparing head-to-head, second line options; in the second line setting, all drugs (regorafenib, cabozantinib, ramucirumab and immunotherapies) were tested after sorafenib; can we extrapolate these results if lenvatinib is used in first line?

\section{Executive summary}

- Hepatocellular carcinoma (HCC) is becoming a major threat in most countries.

- Most patients are seen with advanced stage disease or have progressed to advanced stage despite local or locoregional treatments.

- In 2008 , for the first time in advanced-stage disease, a systemic treatment, sorafenib, demonstrated efficacy when compared with placebo:

- Side effects were frequent but experience and preventive measures improved tolerance and outcomes.

- No biomarkers have been discovered so far, but some clinical subgroups (without extrahepatic spread, hepatitis C virus infection, low neutrophil-to-lymphocyte ratio) derived a greater benefit from sorafenib.

- Some adverse events (dermatologic) are associated with better survival which means that when patients get these side effects, we must try to avoid discontinuation of the drug and insist on local treatment and dose reductions.

- Lenvatinib in first line was noninferior to sorafenib in a large Phase III noninferiority trial, with a larger antitumor effect (response rate, time to progression). Adverse events were as expected and manageable.

- All second-line treatments have been tested versus placebo after sorafenib failure or intolerance:

- Regorafenib, in patients who tolerated sorafenib, significantly improved survival with an acceptable tolerance.

- Cabozantinib has been tested after sorafenib failure or intolerance and improved survival when compared with placebo. In most patients dose reductions were needed due to intolerance but at lower doses, adverse effects were infrequent.

- Ramucirumab, a purely antiangiogenic monoclonal antibody, demonstrated efficacy after sorafenib in the subgroup of patients with high $\alpha$-fetoprotein level. Safety was poor in case of decompensated cirrhosis; in Child-Pugh A patients the safety profile was good.

- Immunotherapies (nivolumab and pembrolizumab) gave impressive results in early Phases (I and II), particularly an excellent safety profile and very long-lasting responses in first and second lines. Results of ongoing Phase III trials are pending.

\section{Financial \& competing interests disclosure}

The authors have no relevant affiliations or financial involvement with any organization or entity with a financial interest in or financial conflict with the subject matter or materials discussed in the manuscript. This includes employment, consultancies, honoraria, stock ownership or options, expert testimony, grants or patents received or pending, or royalties.

No writing assistance was utilized in the production of this manuscript.

Open access

This work is licensed under the Attribution-NonCommercial-NoDerivatives 4.0 Unported License. To view a copy of this license, visit http://creativecommons.org/licenses/by-nc-nd/4.0/

\section{References}

Papers of special note have been highlighted as: $\bullet$ of interest; $\bullet \bullet$ of considerable interest

1. European Association for the Study of the Liver. Electronic address: easloffice@easloffice.eu; European Association for the Study of the Liver. EASL Clinical Practice Guidelines: management of hepatocellular carcinoma. J. Hepatol. 69(1), 182-236 (2018).

- More recent guidelines.

2. Siegel AB, Zhu AX. Metabolic syndrome and hepatocellular carcinoma: two growing epidemics with a potential link. Cancer 115(24), 5651-5661 (2009).

3. Michelotti GA, Machado MV, Diehl AM. NAFLD, NASH and liver cancer. Nat. Rev. Gastroenterol. Hepatol. 10(11), 656-665 (2013).

4. Global Burden of Disease Liver Cancer C, Akinyemiju T, Abera S et al. The burden of primary liver cancer and underlying etiologies from 1990 to 2015 at the global, regional, and national level: results from the Global Burden of Disease Study 2015. JAMA Oncol. 3(12), 1683-1691 (2017).

5. Forner A, Reig M, Bruix J. Hepatocellular carcinoma. Lancet 391(10127), 1301-1314 (2018).

6. Llovet JM, Ricci S, Mazzaferro V et al. Sorafenib in advanced hepatocellular carcinoma. N. Engl. J. Med. 359(4), 378-390 (2008).

-. Seminal paper on sorafenib. 
7. Cheng AL, Kang YK, Chen Z et al. Efficacy and safety of sorafenib in patients in the Asia-Pacific region with advanced hepatocellular carcinoma: a Phase III randomised, double-blind, placebo-controlled trial. Lancet Oncol. 10(1), 25-34 (2009).

8. Raoul JL, Gilabert M, Adhoute X, Edeline J. An in-depth review of chemical angiogenesis inhibitors for treating hepatocellular carcinoma. Expert Opin. Pharmacother. 18(14), 1467-1476 (2017).

9. Kudo M, Finn RS, Qin S et al. Lenvatinib versus sorafenib in first-line treatment of patients with unresectable hepatocellular carcinoma: a randomised Phase III noninferiority trial. Lancet 391(10126), 1163-1173 (2018).

-. Demonstration of efficacy of lenvatinib in hepatocellular carcinoma (HCC).

10. Bruix J, Qin S, Merle P et al. Regorafenib for patients with hepatocellular carcinoma who progressed on sorafenib treatment (RESORCE): a randomised, double-blind, placebo-controlled, Phase III trial. Lancet 389(10064), 56-66 (2017).

-. First demonstration of efficacy in second line.

11. Abou-Alfa GK, Meyer T, Cheng AL et al. Cabozantinib in patients with advanced and progressing hepatocellular carcinoma. N. Engl. J. Med. 379(1), 54-63 (2018).

-. Positive trial with cabozantinib in second line.

12. Zhu AX, Kang YK, Yen CJ et al. REACH-2: a randomized, double-blind, placebo-controlled Phase III study of ramucirumab versus placebo as second-line treatment in patients with advanced hepatocellular carcinoma and elevated baseline alpha-fetoprotein following first-line sorafenib. Presented at: 2018 ASCO Meeting. Chicago, IL, USA May 20 (2018).

13. Zhu AX, Kang YK, Yen CJ et al. Ramucirumab after sorafenib in patients with advanced hepatocellular carcinoma and increased alpha-fetoprotein concentrations (REACH-2): a randomised, double-blind, placebo-controlled, Phase III trial. Lancet Oncol. 20(2), 282-296 (2019).

-. Demonstration of efficacy of ramucirumab in a subgroup of hepatocellular carcinoma (high AFP).

14. El-Khoueiry AB, Sangro B, Yau T et al. Nivolumab in patients with advanced hepatocellular carcinoma (CheckMate 040): an open-label, non-comparative, Phase I/II dose escalation and expansion trial. Lancet 389(10088), 2492-2502 (2017).

- First demonstration of promising data with immunotherapy.

15. Zhu AX, Finn RS, Edeline J et al. Pembrolizumab in patients with advanced hepatocellular carcinoma previously treated with sorafenib (KEYNOTE-224): a non-randomised, open-label Phase II trial. Lancet Oncol. 19(7), 940-952 (2018).

- Interesting data with immunotherapy in second line.

16. Bruix J, Cheng AL, Meinhardt G et al. Prognostic factors and predictors of sorafenib benefit in patients with hepatocellular carcinoma: analysis of two Phase III studies. J Hepatol. 67(5), 999-1008 (2017).

- $\quad$ Predictive and prognostic factors on sorafenib in first line.

17. Reig M, Torres F, Rodriguez-Lope C et al. Early dermatologic adverse events predict better outcome in HCC patients treated with sorafenib. J. Hepatol. 61(2), 318-324 (2014).

18. Rimola J, Diaz-Gonzalez A, Darnell A et al. Complete response under sorafenib in patients with hepatocellular carcinoma: relationship with dermatologic adverse events. Hepatology 62(2), 612-622 (2017).

19. Finn RS. Survival after sorafenib: expect the unexpected. J. Hepatol. 60(2), 243-244 (2014).

20. Shao YY, Wu CH, Lu LC et al. Prognosis of patients with advanced hepatocellular carcinoma who failed first-line systemic therapy. $J$. Hepatol. 60(2), 313-318 (2014).

21. Reig M, Rimola J, Torres F et al. Postprogression survival of patients with advanced hepatocellular carcinoma: rationale for second-line trial design. Hepatology 58(6), 2023-2031 (2013).

22. Ogasawara S, Chiba T, Ooka Y et al. Post-progression survival in patients with advanced hepatocellular carcinoma resistant to sorafenib. Invest. New Drugs 34(2), 255-260 (2016).

23. Ikeda K, Kudo M, Kawazoe $S$ et al. Phase II study of lenvatinib in patients with advanced hepatocellular carcinoma. J. Gastroenterol. 52(4), 512-519 (2017).

24. Kudo M. Lenvatinib in advanced hepatocellular carcinoma. Liver Cancer 6(4), 253-263 (2017).

25. Bruix J, Tak WY, Gasbarrini A et al. Regorafenib as second-line therapy for intermediate or advanced hepatocellular carcinoma: multicentre, open-label, Phase II safety study. Eur. J. Cancer 49(16), 3412-3419 (2013).

26. Finn RS, Merle P, Granito A et al. Outcomes of sequential treatment with sorafenib followed by regorafenib for HCC: additional analyses from the Phase III RESORCE trial. J. Hepatol. 69(2), 353-358 (2018).

27. Kelley RK, Verslype C, Cohn AL et al. Cabozantinib in hepatocellular carcinoma: results of a Phase II placebo-controlled randomized discontinuation study. Ann. Oncol. 28(3), 528-534 (2017).

28. Zhu AX, Finn RS, Mulcahy M et al. A Phase II and biomarker study of ramucirumab, a human monoclonal antibody targeting the VEGF receptor-2, as first-line monotherapy in patients with advanced hepatocellular cancer. Clin. Cancer Res. 19(23), 6614-6623 (2013).

29. Zhu AX, Park JO, Ryoo BY et al. Ramucirumab versus placebo as second-line treatment in patients with advanced hepatocellular carcinoma following first-line therapy with sorafenib (REACH): a randomised, double-blind, multicentre, Phase III trial. Lancet Oncol. 16(7), 859-870 (2015). 
30. Zhu AX, Baron AD, Malfertheiner $\mathrm{P}$ et al. Ramucirumab as second-line treatment in patients with advanced hepatocellular carcinoma: analysis of REACH trial results by Child-Pugh score. JAMA Oncol. (2016).

31. FDA. www.fda.gov/Drugs/InformationOnDrugs/ApprovedDrugs/ucm577166.htm

32. Yoon SE, Hur JY, Lee KK et al. Real-world data on nivolumab treatment in Asian patients with advanced hepatocellular carcinoma. Ann. Oncol. 29(Suppl_8), (2018), ESMO 2018 meeting Munich mdy282.

33. Alsina A, Kudo M, Vogel A et al. Subsequent anticancer medication following first-line lenvatinib: a posthoc analysis from the Phase III REFLECT study in unresectable hepatocellular carcinoma. J. Clin. Oncol. 37, Abstract 371 (2019).

34. Cherny NI, Sullivan R, Dafni U et al. A standardised, generic, validated approach to stratify the magnitude of clinical benefit that can be anticipated from anti-cancer therapies: the European Society for Medical Oncology Magnitude of Clinical Benefit Scale (ESMO-MCBS). Ann. Oncol. 26(8), 1547-1573 (2015).

- Excellent approach to analyze positive results of randomized controlled trials. 